\title{
The bear bones: mosaic ancestry of Macaca arctoides explains divergent baculum morphology
}

Laurie S. Stevison ${ }^{1,}{ }^{*}$, Zachary A. Szpiech ${ }^{1}$, Nick P. Bailey ${ }^{1}$, Taylor E. Novak ${ }^{1}$, Don J. Melnick ${ }^{2, \dagger}$, Ben J. Evans 3 , Jeffrey D. Wall ${ }^{4}$

1Department of Biological Sciences, Auburn University, Auburn, AL 36849, USA

2Department of Ecology, Evolution, and Environmental Biology, Columbia University, 10th floor Schermerhorn Extension, 119th Street and Amsterdam Avenue, New York,NY10027, USA 3Biology Department, Life Sciences Building Room 328, McMaster University, 1280 Main Street West, Hamilton, ON, Canada L8S4K1

${ }^{4}$ Institute for Human Genetics, University of California, San Francisco, California 94143

*Corresponding Author

†Deceased April 18, 2019

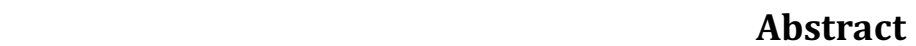

Genital divergence contributes to reproductive barriers between species. Emergence of a novel accessory structure, the baculum, has independently evolved and been lost throughout mammalian evolution, purportedly driven by sexual selection. In primates, the longest recorded baculum belongs to Macaca arctoides, the bear macaque. This species has been proposed to be of homoploid hybrid origin via ancient hybridization between representatives from the fascicularis and sinica species groups. To investigate the evolutionary origins of the bear macaque and its unique morphology, we used whole genome sequences to quantify gene flow and phylogenetic relationships in 10 individuals from 5 species, including the bear macaque $(n=3)$, and two species each from the sinica $(n=3)$ and fascicularis $(n=4)$ species groups. The results of these analyses were concordant, and identified 608 genes in the bear macaque that supported both clustering between M. arctoides and the sinica group (topo2) and had shared derived alleles between species from the two groups. Similarly, 361 genes supported both clustering between M. arctoides and the fascicularis group (topo3) and had shared derived alleles between both groups. Further, sliding window analysis of phylogenetic relationships revealed 53\% of the genomic regions supported placement of $M$. arctoides in the sinica species group (topo2), 16\% supported placement in the 
29 fascicularis species group (topo3), and 11\% supported M. arctoides in a grouping distinct from the

30 sinica and fascicularis groups (topo1). Genomic regions with topo1 were intersected with

31 previously identified QTL for mouse baculum morphology, and 47 genes were found, including five

32 of sixteen major candidate loci that govern mouse baculum variation (KIF14, KIAA0586, RHOJ,

33 TGM2, and DACT1). Although baculum morphology in the bear macaque is diverged from its parent

34 taxa, it most closely resembles that of the fascicularis group. Outliers of shared ancestry from the

35 fascicularis species group located within these same QTL regions overlap with the gene BMP4,

36 which is an important component of the hedgehog signaling pathway that controls gonadogenesis.

37 Two additional outlier genes (one shared with each species group) outside of the baculum QTL are

38 known to interact with BMP4, suggesting this pathway may be involved in baculum morphology in

39 primates. These results highlight how the mosaic ancestry of the bear macaque could explain its

40 unique baculum evolution and collectively contribute to reproductive isolation.

\section{Introductory Paragraph}

In mammals, the baculum has extreme morphological variability, a dynamic evolutionary

43 history characterized by repeated gain and loss, and is often used in species identification. The bear

44 macaque has divergent genital morphology, including the longest baculum among all primates, and

45 is proposed to have evolved via ancient hybrid speciation. Here, population genetic and

46 phylogenomic approaches were used to examine how ancient hybridization in the bear macaque

47 may have shaped this important component of genital morphology. Results demonstrate extensive

48 mosaicism across the genome, which is consistent with ancient genetic contributions from both

49 putative parental taxa. Genetic regions associated with baculum morphology also had mosaic

50 ancestry for several genes, including KIF14 and KIAA0586, major candidate genes for baculum

51 morphology in mice, and BMP4, a developmental gene involved in gonadogenesis. These results 
52 have important implications for how hybridization may have shaped the evolution of reproductive

53 isolation in this unusual species with complex speciation.

\section{Introduction}

For species with internal fertilization, male genital morphology has complex evolution and diverges rapidly1-3. The baculum, or penis bone, is a novel male genital structure in placental mammals that evolved $\sim 145$ million years ago ${ }^{4}$. Since that time, it has been the subject of recurrent adaptation having been gained or lost multiple times in various mammals, including recent loss in Homo sapiens 5 . Despite its remarkable interspecific variation, the penis bone generally does not vary extensively within species ${ }^{6}$, and may be the only physical trait that differs consistently

61 between closely related species, making it a key component in species identification 5 . However, the 62 genetics of morphological variation in this novel trait are not well known. In 2016, a morphometric 63 analysis using micro-CT scans of bacula in mice identified three major quantitative trait loci (QTLs),

64 two that explained $50.6 \%$ of the variation in baculum size, and one that explained $23.4 \%$ of the variation in baculum shape ${ }^{7}$. These regions were further narrowed by differential gene expression in early development of male mice and potential involvement in bone or genital morphogenesis, revealing 16 major candidate loci (see Table S3 in ref $^{7}$ ). Sequence divergence to rats reduced this list even further to four major candidate genes, all of which have orthologs in primates (PTPRC, KIAA0586, ASPM, and KIF14)7.

Among primates, the bear macaque, Macaca arctoides has the longest baculum $\left(4-6 \mathrm{~cm}^{8}\right)$,

71 even when corrected for body weight ${ }^{8,9}$. This species is perhaps the most unusual among macaques,

72 distinguished not only by its extreme baculum length, but also by divergent genital morphology in

73 both males and females (Figure S1), a unique single-mount ejaculatory mating behavior, whitish

74 pelage in newborns, and a bald forehead and cheeks ${ }^{8}$. One possible explanation for the origin of the

75 bear macaque baculum is that natural selection favored the evolution of prezygotic barriers to 
hybridization via a mechanical "lock and key" mechanism"10. Interestingly, however, this dramatic

77 derived genital morphology nonetheless may have been influenced by, or evolved despite, extensive genetic exchange with other macaque species. Early molecular work revealed phylogenetic incongruence between mitochondrial and Y-chromosomal genealogies for $M$. $\operatorname{arctoides}^{10,11}$. Mitochondrial markers place M. arctoides as sister to the fascicularis species group, whereas autosomal markers place it as sister to the sinica species group ${ }^{12-15}$. However, both morphological and Y-chromosomal trees support placement in the sinica group ${ }^{11,15,16}$ (see Table S1 in ref $^{14}$ for review). This incongruence was inferred to be consistent with ancient hybridization

84 between the ancestor of the fascicularis species group and a sinica species group member (an

85 ancestor of either M. assamensis or M. thibetana) ${ }^{10}$. This hypothesized hybrid origin is diagrammed in Figure 1B. animals 17,18 . Though expected to be a rare speciation mechanism relative to "regular" bifurcating speciation, diverse taxa such as birds (Passer italiae ${ }^{19}$ ), butterflies (Heliconius heurippa ${ }^{20}$ ) and yeast

91 (Saccharomyces paradoxus ${ }^{21}$ ) are thought to have evolved via HHS. Recent mitochondrial

92 phylogenetics have shown that $M$. arctoides is more closely related to M. mulatta as compared to $M$.

93 fascicularis ${ }^{14,22}$, indicating introgression may have occurred after the split of these two species,

94 which is estimated to be 1.68 mya ${ }^{14,15,23}$ (Figure 1B). This suggests an alternative evolutionary

95 scenario to the previously proposed model for the origin of $M$. arctoides by hybrid speciation; this

96 alternative is also presented in Figure 1B.

97 Recent analysis of macaque genomes support introgression between M. arctoides and

98 members of the fascicularis and sinica species groups ${ }^{14}$, both of whom it overlaps with

99 geographically (Figure 1C). The baculum in M. arctoides is more than double the average length of 100 sinica species group taxa and nearly four times as long as fascicularis species group taxa9,24. These 
101 sister species groups are estimated to have split $\sim 3$ million years ago, with hybridization to

102 produce $M$. arctoides purportedly occurring $\sim 1.8$ million years ago ${ }^{14}$, during which glacial periods

103 during the Pleistocene were associated with reduced expanse of Southeast Asian forests into

104 multiple refugia. Ecological isolation is a common feature to other scenarios of HHS 25 , and may have

105 contributed to the extensive interspecific hybridization that gave rise to the unique macaque

106 species, M. arctoides. Y-chromosomal data suggests that interbreeding between ancestors of the

107 fascicularis and sinica groups was driven by sinica males. Available evidence suggests that this

108 interbreeding subsided $\sim 1.5$ mya $^{10}$, followed by little interbreeding until the present 8 .

109 Here, we investigated the role hybridization played in shaping the unusual genital

110 morphology of M. arctoides. Because a major criteria for HHS26,27 is that reproductive isolation (RI)

111 be a direct byproduct of hybridization (but see ${ }^{28}$ ), we focused especially on genetic regions that are

112 associated with morphological variation and candidate genes in mice5. Phylogenomic and

113 population genetic approaches were used on several extant macaque species (M. arctoides; the

114 sinica group species M. thibetana and M. assamensis; and the fascicularis group species $M$.

115 fascicularis and M. mulatta). Also, outlier regions were examined within the bear macaque genome

116 with alternate alleles from the two parental genomes and regions where the bear macaque differs

117 from both parents. Despite its divergent morphology $8,9,29$, the baculum of M. arctoides is more

118 similar to fascicularis species ${ }^{8}$, making these outliers of particular interest.

\section{Results}

Genomic analysis of five macaque species revealed a mosaic of evolutionary ancestry with

121 respect to both putative parental species; this generated novel allelic combinations at multiple loci

122 throughout the genome, including several genes associated with baculum morphology. These

123 analyses used newly sequenced whole genome samples from $M$. arctoides (20X coverage) and $M$.

124 assamensis (13X), and 8 publicly available genomes ranging in coverage from 4-49X (median 32X; 
125 Table 1). While not directly investigated here, these data will be useful for addressing questions

126 related to the putative hybrid origin of this species in combination with targeted simulations (see

127 Discussion).

128 Of the heterozygous sites, the majority are unique to each species group (Figure 1D). $M$.

129 arctoides shares the most variants with the sinica species group, M. assamensis and M. thibetana,

130 consistent with taxonomic placement in this species group ${ }^{30}$. However, the two-way intersection

131 between $M$. arctoides and M. thibetana had the fewest number of shared heterozygous sites, which

132 is likely due to the low overall heterozygosity in the M. thibetana sample (Figure 1D; Table 1). $M$.

133 assamensis had the largest number of heterozygous sites ( 4.7 million; Table 1; Figure 1D). This

134 high variability is consistent with the demographic results from smc++, which indicated population

135 size expansion around 10,000 years ago in M. assamensis but not M. thibetana and M. arctoides

136 (Figure 1E). Consistent with this, PSMC analysis in a previous study showed M. assamensis has a

137 higher overall effective population size beginning 250 kya compared to M. arctoides and other

138 sinica species (see Figure 3B in ${ }^{14}$ ).

Extensive Mosaicism of the M. arctoides Genome

The four-taxon test ${ }^{31-33}$ results supported that the M. arctoides genome has a mosaic of

141 shared ancestry with both the sinica and fascicularis species groups (Figure 2), which is consistent

142 with extensive introgression from both species groups. Though it is worth noting that windows

143 with shared ancestry could also be due to incomplete lineage sorting between these taxa ${ }^{23}$. The

144 genome-wide average $f_{d M^{34,35}}$ value was -0.113 , ranging from -0.69 (supporting shared ancestry

145 with the sinica group) and 0.55 (supporting shared ancestry with the fascicularis group) in $50 \mathrm{~kb}$

146 sliding windows. This sliding window analysis was repeated with several window sizes (Figure S2),

147 and all had a similar genome-wide mean $f_{d M}$. In a previous study, estimates of $D$ were similar to the

$148 f_{d M}$ estimates here (see Table S8 in $^{14}$ ). 
150 chromosome to have significantly lower mean $f_{d M}$ estimate $(-0.15)$, with a statistical difference

151 between the two distributions (Figure S3). There was a non-significant negative relationship

152 between chromosome length and $f_{d M}$, but the X-chromosome has an intermediate length (149 $\left.\mathrm{Mb}\right)$

153 compared to the autosomes (mean=134 Mb). Thus, the lower $f_{d M}$ estimate on the $\mathrm{X}$ is not likely

154 attributed to the length of the chromosome.

155 Phylogenetic relationships among the species groups were evaluated via the software

156 Twisst ${ }^{36}$ (topology weighting by iterative sampling of subtrees). This method quantifies

157 phylogenetic relationships across the genome and returns weights in sliding windows (Figure 1F).

158 The topology weights were used to define a "majority topology" as having two-thirds or more of the

159 sum of weights in any region. Windows that did not meet this criterion were re-classified as

160 "unresolved". This analysis showed the proportion of the genome that supported topo2, which

161 groups $M$. arctoides with the sinica group, as 52.64\% (95\% bootstrap CI [52.02\%, 53.28\%]).

162 However, a significant portion, 15.70\% (95\% bootstrap CI [15.40\%, 16.01\%]), of the genome

163 supported topo3, which groups M. arctoides with the fascicularis species group. Interestingly,

$16411.07 \%$ (95\% bootstrap CI [10.84\%, 11.31\%]) of the genome supported $M$. arctoides clustering in a

165 group by itself (topo1). The remaining 20.59\% (95\% bootstrap CI [20.18\%, 21.01\%]) of the genome

166 was categorized as unresolved. Still, the $15.70 \%$ of the genome that supports topo3 is a rather large

167 fraction of the genome considering this is an ancient hybridization event. Similar to our four-taxon

168 results, this distribution of support for each of the three possible topologies is different on the $\mathrm{X}$

169 chromosome, with an excess of topo 2 on the X (70.89\%, 95\% bootstrap CI [67.49\%, 74.18\%]). One

170 possible explanation for this difference relates to incomplete lineage sorting which is expected to be

171 lower on the X-chromosome as compared to the autosomes, owing to the lower effective population

172 size of the $\mathrm{X}$.

173 In addition to examining broader patterns of shared ancestry in this species, outliers of 
174 shared ancestry with each species group and the M. arctoides genome were specifically scrutinized

175 (see below). This set of regions were intersected with the Ensembl gene predictions downloaded

176 from University of California Santa Cruz (UCSC) Genome Browser, resulting in 371 genes with the

177 highest ancestry from the fascicularis group, and 608 genes with the highest ancestry from the

178 sinica group. These results were highly concordant with the majority topology for each of the two

179 possible groupings between $M$. arctoides and the parental taxa. The intersection resulted in no

180 reduction of the 608 sinica-shared genes, whereas the fascicularis-shared genes were reduced to

181363 from 371. This set of 971 genes were uploaded to the Panther Database. The enrichment gene

182 terms for GO Biological Processes revealed three GO terms enriched from fascicularis species group

183 and 24 from sinica species group (Table S5). A surprising result was that there were three

184 neuronal-related gene categories for ancestry shared between sinica and M. arctoides (see below

185 and Discussion).

Intersection of Regions Associated with Baculum Morphology with Topo1 Regions

Next, the phylogenomic results were intersected with the mouse baculum QTL to investigate defining the majority topology was increased to require $100 \%$ of the weights into topo1, which

192 reduced this to $4.5 \%$ of the genome. The regions from the Twisst analysis were extracted and 193 intersected with the known Ensembl gene annotations, resulting in 4175 genes. A GO Analysis of 194 these genes revealed enrichment of gene categories involved in developmental processes, immune 195 system processes, and metabolic processes (Figure 4). There were also gene categories involved in 196 reproduction, reproductive processes, behavior, and pigmentation, which have particular 197 importance in M. arctoides given its phenotypic changes in these traits. 
This gene list was intersected with homologous chromosome regions in rheMac8 to the

199 three major mouse baculum QTL, leaving 47 genes. Five of these genes (TGM2, KIF14, RHOJ, DACT1,

200 and KIAA0586) were listed in Table S3 of $\operatorname{ref}^{7}$ as the 16 major candidate loci that overlap QTL

201 regions for baculum morphology in mice. Two of the five genes, KIF14 and KIAA0586, also had a

202 high sequence divergence between mouse and rat, thus being considered as two of four major

203 candidate loci in explaining the genetic basis of mouse baculum size variation ${ }^{7}$. Similarly,

204 divergence between M. arctoides and the sinica and fascicularis species group members was also

205 high for KIF14 (Sin-Arc $F_{S T}=0.45$; Fas-Arc $\left.F_{S T}=0.61\right)$ and KIAA0586 (Sin-Arc $F_{S T}=0.38$; Fas-Arc $F_{S T}$

$206=0.52)$ compared to the genome-wide averages (Sin-Arc $F_{S T}=0.33 ;$ Fas-Arc $\left.F_{S T}=0.43\right)$. Ten of the

20747 genes also had missense mutations between the mouse strains used for the QTL mapping (see

208 Table S2 in ref $^{7}$ ). Two of the 10 genes with missense mutations also had high F F: DDX59 (Sin-Arc

$209 \mathrm{~F}_{\mathrm{ST}}=0.40 ;$ Fas-Arc $\mathrm{F}_{\mathrm{ST}}=0.52$ ) and SOGA1 (Sin-Arc $\mathrm{F}_{\mathrm{ST}}=0.38 ;$ Fas-Arc $\mathrm{F}_{\mathrm{ST}}=0.51$ ).

210 For the two top candidate genes, KIF14 and KIAA0586, M. arctoides differed from all other

211 lineages at 15 and 13 nucleotide positions, respectively. M. arctoides had two fixed and six

212 heterozygous non-synonymous mutations in the protein-coding region of KIAA0586 and one fixed

213 and one heterozygous non-synonymous mutation in KIF14. Neither of the fixed differences were

214 located in any known functional domains. One of the other five genes (DACT1) had a premature

215 stop codon fixed in M. arctoides; however, the impact to overall function is unclear (but see

216 Discussion).

217 For fixed non-coding variants and variants immediately upstream or downstream of each

218 gene, $100 \mathrm{bp}$ regions surrounding variants for each allele were input into the online tool TomTom ${ }^{38}$

219 to identify potential binding sites for transcription factors (Table S8). One of these SNPs suggested

220 that CTCF binds to the M. arctoides allele of KIF14, but not any of the others (Figure 3A). The

221 ENCODE Transcription Factor Target dataset as accessed through Harmonizone ${ }^{39}$ suggests that

222 CTCF is a known transcription factor for KIF14. 


\section{Gene Flow in Regions Associated with Baculum Morphology}

To understand how the mosaic ancestry of the M. arctoides genome contributed to its

225 extreme baculum morphology and subsequent RI, the outliers of shared ancestry were also

226 intersected with regions in rheMac8 homologous to the mice baculum QTLs. ANOVA indicated the

227 distribution of $f_{d M}$ scores in these regions were significantly different from the rest of the genome.

228 Surprisingly, these regions were more negative, indicating more shared ancestry with sinica

229 member species than fascicularis group species (Figure S4), which is the opposite of the

230 expectation based on morphology.

Because the baculum of M. arctoides is more similar to members of the fascicularis species group than the sinica group, the fascicularis-shared introgression outlier regions (shown in blue in

233 Figure S4) were further examined. Of 363 genes identified genome-wide, three genes were located

234 within one of the three QTL regions on chromosome 7 (Figure S5; Supplementary Methods and

235 Results). One of these genes, Bone Morphogenetic Protein 4 (BMP4), is a key gene in the hedgehog

236 signaling pathway ${ }^{40}$, and has a role in gonadal development ${ }^{41,42}$. As expected for a major

237 developmental gene, no coding differences were observed. However, there were two variants in the

238 3' UTR of BMP4, one of which matched topo3, where M. arctoides and fascicularis species had a

239 shared allele (Figure 3C; Figure S6A). Using TomTom as described above, differential binding was

240 predicted for variants at each SNP which identified three genes known to interact with BMP4: Six1,

241 Tead1, and Tead3. Six1 deficiencies lead to reduced BMP4 expression ${ }^{43}$ and affect spatial

242 expression patterns ${ }^{44}$. Similarly, Tead proteins are a family of transcription factors that mediate

243 transcription and cell migration of BMP4 via the TAZ component of the Hippo pathway ${ }^{45}$. Based on

244 the alleles present, the lack of Six1 binding and the added binding of Tead1 and Tead3 would

245 predict an increase in expression of BMP4 in M. arctoides and fascicularis group species as

246 compared to sinica group species. 


\section{Characterization of Outlier Genes Explain Other Phenotypes Unique to M. arctoides}

As mentioned earlier, M. arctoides has other traits (e.g. ontogenetic coloration, prolonged

249 intromission with a postejaculatory "pair-sit", etc ${ }^{8}$.) that make it unusual among macaques. Of the

250971 genes identified as shared ancestry outliers, a stringent set of 146 genes, 51 fascicularis and 95

251 sinica origin genes were identified (see Supplementary Methods and Results) and manually

252 curated. The seven manually curated categories had the following number of genes: 11 bone, 12

253 reproductive, 7 sensory, 8 immune and 6 skin/fur. These highly represented groups had: 55 cellular

254 functions and 55 neural functions (Table S6). Some genes were in more than one category. The two

255 highly represented groups corresponded well with the prevalence of neuronal-related gene

256 categories from the GO analysis for sinica outliers. One possibility for this neural enrichment is that

257 interactions between the fascicularis-shared mtDNA and the mostly sinica-shared nucleus may

258 have led to mitonuclear incompatibilities between these species (see Discussion).

A moderate number of bone, reproductive, and skin/fur genes is unsurprising based on the

260 unique biology of M. arctoides. Notably, two known interactors with BMP4 (DYNC2LI1 and

261 CTDSPL2) were included in this stringent gene list. DYNC2LI1 exhibited shared ancestry between

$262 M$. arctoides and fascicularis $\left(f_{d M}=0.79\right)$ whereas CTDSPL2 exhibited shared ancestry between $M$.

263 arctoides and sinica $\left(f_{d M}=-0.82\right)$. In humans, DYNC2LI1 mutations are associated with lethal

264 skeletal diseases and have been found to impair the Hedgehog pathway ${ }^{46}$. CTDSPL2 phosphorylates

265 various Smad proteins, which are transcriptional regulators directly involved with the BMP

266 pathway. CTDSPL2 knock-down experiments in mice lead to increased expression of BMP targeted

267 genes ${ }^{47}$.

TomTom was used to examine putative binding motifs associated with the differing alleles

270 fascicularis/outgroup had 16 fixed differences between them and 5 heterozygous sites. Of these,

271 only one transcription factor seemed to be directly related to regulation of CTDSPL2. Specifically, 
overexpression of XBP1 in mouse cells up-regulates CTDSPL2448. TomTom results suggested that

273 XBP1 binds to the shared M. arctoides/sinica allele of CTDSPL2 but not the shared

274 fascicularis/outgroup allele (Figure 3B). The DYNC2LI1 gene had 12 fixed differences between $M$.

275 arctoides/fascicularis and the sinica/outgroup, one resulting in a non-synonymous mutation (c.

276 422G>A; p. Arg>Lys141). TomTom results suggest that this SNP changes the binding affinity of

277 RBFOX2 protein, which regulates splicing events ${ }^{49}$. TomTom predicted binding of RBFOX2 to the

278 allele shared by M. arctoides and fascicularis species (Figure 3C). See Table S8 for detailed

279 characterization of TomTom results for each SNP.

\section{Discussion}

\section{A patchwork of ancestral origins for the baculum in the bear macaque}

The gene analysis of alleles unique to the bear macaque lineage revealed 47 genes that overlapped three QTL regions associated with baculum morphology in mice. Two of these genes are major candidate genes for baculum size, KIF14 and KIAA05867 , the latter of which has several nonsynonymous mutations. Three other genes were on a longer list of 16 candidate genes, one of which (DACT1) had a nonsense mutation c. 1317G>A; p. Trp439* fixed in M. arctoides. DACT1 is part of the Wnt signaling pathway, interacts with dishevelled (DVL), and is associated with Townes-

Brocks syndrome 2 (TBS2; MIM\# 617466). Interestingly, a heterozygous nonsense mutation c.

1256G>A; p. Trp419* in DACT1 in humans has been shown to have a variety of phenotypes including genitourinary malformations. These malformations include hypospadias ${ }^{50}$, a birth defect where the urethral opening is on the underside of the penis, which is also characteristic of $M$. arctoides male genital morphology ${ }^{8}$. 
clustering of the bear macaque with the sinica species group, and instead morphologically

297 resembles members of the fascicularis species group ${ }^{8}$. Consistent with these morphological

298 similarities, one of 363 fascicularis-sourced introgression outliers overlaps with a QTL associated

299 with variation in baculum size in mice. This gene, BMP4, is a major regulator of bone formation and

300 gonad development ${ }^{40,42}$. Two SNPs in the 3' UTR have alternative binding motif alleles that suggest

301 regulatory changes in this gene that would result in upregulation of BMP4 in M. arctoides as

302 compared to sinica species (Figure 6C). Still, functional validation is needed to ascertain whether

303 this genomic variation is coupled with differences in expression intensity, location, or timing during

304 development.

305 Additionally, among the 47 genes overlapping the mouse QTLs from topo1, one gene with 306 notably high $\mathrm{F}_{\mathrm{ST}}$ was the gene BMP/Retinoic Acid Inducible Neural Specific 3 (BRINP3; Sin-Arc $\mathrm{F}_{\mathrm{ST}}=$

3070.48 , Fas-Arc $\mathrm{F}_{\mathrm{ST}}=0.60$ ). There were two other genes in the outlier analysis that are known to

308 interact with BMP4 and bone development, pointing to possible rewiring of this important

309 developmental pathway in the M. arctoides genome. Although this pathway was not identified in the

310 mouse baculum morphology study, it was mentioned as a likely candidate worthy of investigation.

311 It is also possible that this pathway is involved in baculum morphology in primates, but not in mice.

312 Future work should focus on these genes to understand the evolution of baculum morphology in $M$.

313 arctoides.

\section{Complex speciation of the bear macaque}

315 Macaques are a diverse primate genus with several examples of purported complex

316 speciation $10,11,50$. Although mtDNA suggests that introgression with the fascicularis group should be

317 driven by M. mulatta ${ }^{14,22}$, when considering these two species separately, the autosomal estimates

318 of $f_{d M}$ are largely similar (Table S3). One possible explanation for this is that the sample used for $M$.

319 fascicularis is Vietnamese in origin and therefore has extensive gene flow from M. mulatta ${ }^{51}$. Though 
320 it is worth noting that there are 1.6 times more shared heterozygous sites between M. arctoides and

321 M. mulatta than between M. arctoides and M. fascicularis (Figure 1D). We also found 11.07\% of

322 genomic regions support a clustering of M. arctoides separate from other macaque species.

323 Although several taxonomic surveys have suggested the placement of M. arctoides into its own

324 species group $30,52,53$, recent genome surveys suggest that sequencing of additional sinica species

325 members will be useful in making this determination ${ }^{54,55}$.

326 While genomic mosaicism is a key feature of hybrid species, it is also a ubiquitous feature of 327 admixture more generally. For example, in swordtail fish, there is genomic mosaicism that resembles 328 hybrid speciation, although it is not consistent with other information from this species $26,27,56$. 329 Similarly, up to $15 \%$ of the genome supports clustering of $M$. arctoides with fascicularis species. This 330 finding, though compelling, is also not uncommon in other taxa that do not have hybrid speciation.

331 For example, in human-chimp-gorilla comparisons, $\sim 30 \%$ of the human genome does not support

332 the species tree ${ }^{57}$. In principle, an even contribution from both parents to a hybrid genome is possible,

333 but this is often not the case in HHS (e.g., Heliconius ${ }^{20}$ ). Here, we observed a significant deviation

334 from a 50-50 split of ancestry from the two groups, which could be due to a combination of proposed

335 recent admixture with sinica group taxa ${ }^{10}$ and differences in ancestral populations sizes of the 336 putative parental taxa.

337 Here, we also found higher shared ancestry between M. arctoides and sinica species group

338 taxa on the X-chromosome as compared to the autosomes, which is consistent with either (1)

339 higher introgression from the sinica group on this chromosome compared to the autosomes, (2)

340 lower effective population size of the X-chromosome compared to the autosomes and/or (3)

341 increased selection against introgression on the $\mathrm{X}$ chromosome (which is qualitatively consistent

342 with Haldane's rule). The latter possibility is interesting in light of a recently proposed

343 mitochondrial capture scenario via reduced fitness of male hybrids ${ }^{14}$. Mitochondrial capture could

344 either have followed M. arctoides split from sinica, or it could have been simultaneous with an HHS 
origin (Figure 1B). While there are recent statistics that explicitly test for HHS ${ }^{58}$, these have a

restrictive set of assumptions that may not apply to this system (e.g., subsequent gene flow with

347 parental taxa). Therefore, a more explicit set of simulations of various speciation scenarios would

348 need to be conducted to determine the timing of ancient introgression from the two parental

349 genomes.

\section{Unanticipated enrichment of introgression outliers with neurological function}

GO terms in the introgression outliers from sinica and M. arctoides origin analysis revealed a

352 large number of genes involved in neurological function that were noticeably absent in the fascicularis outliers. A possible explanation is that the mismatch in sinica-sourced mitonuclear genes

354 could lead to mitochondrial dysfunction, which could contribute to neural malfunction and disease.

355 For example, disease-causing mutations in mitochondrial aminoacyl tRNA-synthetases in humans 356 predominantly result in neurological disorders ${ }^{59}$. This is believed to be due to the generally high-

357 energy demand of primate brains and may necessitate coevolution of mitonuclear and mitochondrial

358 genes 60,61 . Moreover, at least two of the sinica-sourced neurological genes characterized here, GFM2

359 and SOD2, are known to be involved in mitochondrial disorders exhibiting neurological effects ${ }^{62,63}$.

360 Additionally, hybridization scenarios exhibiting mismatch between mitochondrial and mitonuclear

361 genes have been discovered in a variety of systems ${ }^{64-66}$. A comprehensive analysis of the potential for

362 mitonuclear incompatibilities in the evolution of $M$. arctoides could also shed light on the evolution

363 of the bear macaque.

\section{Future Directions}

This study has focused on the baculum based on its extreme length in M. arctoides, which is proposed to play a role in reproductive isolation among species However, we recognize that the

367 baculum is certainly not the only barrier to reproduction between M. arctoides and its parental taxa. 368 We anticipate there are other regions of the genome that have contributed to speciation ${ }^{67}$. For 
example, there have been compensatory changes in female reproductive morphology ${ }^{29}$ that are equally important in the mechanical barrier between these taxa (Figure S1). Additionally, changes

371 in their mating behavior also contribute to differences between M. arctoides and its parent taxa ${ }^{9}$.

372 Therefore, as the genetic basis of such traits become known, they can be further investigated.

374 However, due to the limited number of publicly available whole genome samples (now three with the

375 present study), the conservation status of this species, and the ethical and logistical barriers to 376 experimental research with primates, it is challenging to make functional insights into its evolution.

377 Additional WGS data from more individuals of this species would allow for a better characterization 378 of selection/adaptation within this lineage. Additionally, more WGS samples would be useful in 379 examining the distribution of introgression haplotype lengths to pinpoint the timing of events in this system that would better clarify when hybridization from each parent took place.

\section{Methods}

\section{Samples and Genome Sequencing}

One female each from M. arctoides (Malaya) and M. assamensis (A20) were sequenced.

384 These two samples were multiplexed and sequenced on one lane of sequencing for initial quality 385 assessment. They were then subsequently run on one additional lane of sequencing each for a final

3861.5 lane per sample. Sequencing was done at UCSF Medical Center Sequencing Facility on a Illumina 387 HiSeq 2000 machine. Each run was assigned as different read groups to be treated as independent 388 in the variant calling workflow. The raw read data have been deposited in NCBI sequence read 389 archive and are available via the accession PRJNA622565. 
1). Additional sample details can be found in the corresponding publications. These were each downloaded from NCBI SRA using sratoolkit version 2.8.1 with options to split data into read pairs

394 and in the original format 68 . Because M. thibetana was sequenced on an older platform, we used 395 seqtk ${ }^{69}$ to convert from Q64 to Q33. Additionally, SRA files from multiple lanes as indicated in the

396 fastq header were split for independent alignment to the reference genome facilitating the

397 definition of separate read groups to be treated as independent in the variant calling workflow.

\section{Alignment, genome analysis, and variant calling}

Raw fastq files from all samples were aligned to the reference genome rheMac8 (NCBI

400 Accession: PRJNA214746). The masked reference genome was downloaded from UCSC. GATK best

401 practices were followed to obtain high quality variant sites (see Supplementary Methods). Briefly,

402 reads were aligned to the reference genome, duplicates were marked, indels were realigned, base

403 quality scores were recalibrated, variants were called, and variant quality scores were recalibrated.

404 Samples from each species were processed independently and merged into a final callset. The

405 baboon reference genome was added to the variant files via two-way genome alignment and 406 custom scripts. These final filtered files for each chromosome with the baboon information are

407 freely available and were used for all subsequent analyses.

\section{Four-taxon test for introgression}

To analyze patterns of introgression in sliding windows, a modified four-taxon test was

410 used (Figure 2). Rather than separately analyzing gene flow between $M$. arctoides and each parental

411 species group, as has been done previously14, we relied on the modified statistic $f_{d M}$, which is better

412 at comparing introgression from both groups ${ }^{35}$. Specifically, hybridization between $M$. arctoides

413 (P3) was tested with members of both the sinica species group (P1) and the fascicularis species

414 group (P2), using Baboon as an outgroup. Calculations were done using scripts available on

415 github 33,34 . To select the appropriate window size for the analysis, a series of different sized 
windowed analyses were conducted. $f_{d M}$ was computed for overlapping windows of size $5 \mathrm{~kb}, 25 \mathrm{~kb}$,

$41750 \mathrm{~kb}, 100 \mathrm{~kb}, 500 \mathrm{~kb}$ and $1000 \mathrm{~kb}$, with a step size of $20 \%$ of each window size. The minimum

418 number of sites per window size was based on the distribution of sites available in the callset at

419 each bin size. Specifically, a minimum number of sites of 10, 50, 100, 200, 1000, and 2000,

420 respectively, per window size were used. Because it is recommended to have at least 100 sites per

421 window $34,50 \mathrm{~kb}$ sliding windows were selected and are shown in Figure 2 as a heatmap across the

422 genome. Results from the additional bin sizes are shown in Figure S2. This analysis was repeated

423 with individual taxa from each species group (Table S3).

\section{$424 \quad$ Topology weighting}

To analyze the weights of different phylogenetic topologies across the genome, the software

426 Twisst was used to compare the weights of different species topologies throughout the genome ${ }^{36,70}$.

427 First, data used for the introgression analysis were pruned to make sure that each group had at

428 least one member represented in the genotype data. Next, neighbor joining trees were constructed

429 in sliding windows of 50 SNPs each across the genome using Phyml from scripts available on

430 github ${ }^{34}$. This generated newick formatted tree files that were used as input for Twisst.

The topology weighted outputs were further explored by collapsing adjacent intervals with

432 the same majority topology supported. Since there were three possible topologies, the majority

433 topology was defined as any one topology that had at least two-thirds of the total weight values.

434 Any intervals without a majority topology were labeled as "unresolved". If adjacent intervals

435 supported the same majority topology, they were collapsed and split into the three major

436 topologies. These were intersected with gene regions from the Ensembl annotations for rheMac8

437 downloaded from UCSC.

438 Bootstrap confidence intervals for topo1, topo2, topo3, and unresolved proportions were

439 computed by resampling 2,792 1Mbp intervals along the genome (149 when considering only

440 chromosome X) for each of 10,000 replicates and taking the $2.5 \%$ and $97.5 \%$ quantiles. 


\section{Demography Analysis using smc++}

The method smc $++{ }^{71}$ was used, which uses whole genome sequencing data across multiple

443 samples and does not require phased data. All sequences were masked at the start of a

444 chromosome up to the first genotype call, at the end of a chromosome from the last genotype call,

445 and all rheMac8 assembly gaps. Then, the estimate module was used to estimate population size

446 history with an assumed mutation rate of $2.5 \mathrm{e}-8^{14}$ and automatic selection of inference timepoints.

447 When generating population size trajectory plots, a generation time of 6 years ${ }^{14}$ was used.

\section{Functional Analysis of Targeted and Outlier Regions}

A major criteria of homoploid hybrid speciation is evidence that RI evolved as a by-product

of hybridization ${ }^{26}$. To investigate this directly, regions associated with morphological variation in

451 the baculum identified in mice ${ }^{7}$ were targeted. The UCSC tool liftover ${ }^{72}$ was used to convert

452 coordinates of the three QTL from the mouse study from mm10 to rheMac8.

454 taxa were extracted from the sliding window results (see Supplementary Methods). These regions

455 were further intersected with gene lists from the Twisst analysis. Specifically, sinica outliers of

456 shared ancestry were intersected with regions where the majority topologies supported $M$.

457 arctoides clustering with sinica species. Similarly, fascicularis outliers of shared ancestry were

458 intersected with regions where the majority topologies supported $M$. arctoides clustering with

459 fascicularis species. These intersected gene lists (Table S4) were used to conduct a GO Analysis

460 using Panther ${ }^{73}$ (Table S5).

\section{Acknowledgements}


464 GM115433 (to JDW). This work was made possible in part by a grant of high performance

465 computing resources and technical support from the Alabama Supercomputer Authority. BJE was

466 supported by a grant from the Natural Science and Engineering Research Council of Canada

467 (RGPIN-2017-05770). We thank members of the Stevison lab for helpful discussions regarding

468 the analysis and results, with particular thanks to Stephen Sefick. We thank Simon Martin both for

469 having a public github repository of high quality, but also for responsiveness regarding inquiries in

470 use of his code. We thank several researchers in attendance at the 2016 Speciation GRC, Evolution

471 2017, and PEQG 2018 for helpful discussions regarding this work. We thank Wendy Hood, Ken

472 Halanych, and Geoff Hill for feedback on early drafts of the manuscript.

\section{Author Contributions}

LSS, JDW, DJM, and BJE conceived of the project. DJM supplied the samples and JDW paid for

475 sequencing. LSS executed methods/analyses with substantial input from JDW and BJE, and wrote

476 the draft manuscript. BJE and DJM significantly aided the interpretation of results. ZAS conducted

477 VQSR and SMC++ analyses. NPB and TEN conducted manual curation of gene outliers and TomTom

478 on baculum alleles. All authors were involved in editing and revision of the final draft.

\section{References}

480 1. Langerhans, R. B., Anderson, C. M. \& Heinen-Kay, J. L. Causes and Consequences of Genital

$481 \quad$ Evolution. Integr. Comp. Biol. 56, 741-751 (2016).

482 2. Klaczko, J., Ingram, T. \& Losos, J. Genitals evolve faster than other traits in Anolis lizards. J. Zool.

$483 \quad 295,44-48(2015)$.

484 3. Eberhard, W. G. Evaluating Models of Sexual Selection: Genitalia as a Test Case. Am. Nat. 142,

$485 \quad 564-571(1993)$.

486 4. Brindle, M. \& Opie, C. Postcopulatory sexual selection influences baculum evolution in primates 487 and carnivores. Proc. R. Soc. B Biol. Sci. 283, 20161736 (2016). 
5. Carosi, M. \& Scalici, M. Baculum (Os Penis). in The International Encyclopedia of Primatology 1-5 (American Cancer Society, 2017). doi:10.1002/9781119179313.wbprim0214.

6. Ramm, S. A., Khoo, L. \& Stockley, P. Sexual selection and the rodent baculum: an intraspecific study in the house mouse (Mus musculus domesticus). Genetica 138, 129 (2009).

7. Schultz, N. G. et al. The Genetic Basis of Baculum Size and Shape Variation in Mice. G3 GenesGenomesGenetics 6, 1141-1151 (2016).

8. Fooden, J. The bear macaque,Macaca arctoides: a systematic review. J. Hum. Evol. 19, 607-686 (1990).

9. Dixson, A. F. Observations on the evolution of the genitalia and copulatory behaviour in male primates. J. Zool. 213, 423-443 (1987).

10. Tosi, A. J., Morales, J. C. \& Melnick, D. J. Paternal, maternal, and biparental molecular markers provide unique windows onto the evolutionary history of macaque monkeys. Evol. Int J Org Evol. 57, 1419-35 (2003).

11. Tosi, A. J., Morales, J. C. \& Melnick, D. J. Comparison of Y Chromosome and mtDNA Phylogenies Leads to Unique Inferences of Macaque Evolutionary History. Mol. Phylogenet. Evol. 17, 133144 (2000).

12. Li, J. et al. Comparative Genome-Wide Survey of Single Nucleotide Variation Uncovers the Genetic Diversity and Potential Biomedical Applications among Six Macaca Species. Int. J. Mol. Sci. 19, (2018).

13. Li, J. et al. Phylogeny of the macaques (Cercopithecidae: Macaca) based on Alu elements. Gene 448, 242-249 (2009).

14. Fan, Z. et al. Ancient hybridization and admixture in macaques (genus Macaca) inferred from

511 15. Jiang, J. et al. Mitochondrial Genome and Nuclear Markers Provide New Insight into the Evolutionary History of Macaques. PLoS ONE 11, (2016). 
513 16. Fan, P. et al. Phylogenetic position of the white-cheeked macaque (Macaca leucogenys), a newly

514 described primate from southeastern Tibet. Mol. Phylogenet. Evol. 107, 80-89 (2017).

515 17. Mallet, J. Hybrid speciation. Nature 446, 279-283 (2007).

516 18. Mavárez, J. \& Linares, M. Homoploid hybrid speciation in animals. Mol. Ecol. 17, 4181-4185

517 (2008).

518 19. Elgvin, T. O. et al. The genomic mosaicism of hybrid speciation. Sci. Adv. 3, e1602996 (2017).

519 20. Jiggins, C. D., Salazar, C., Linares, M. \& Mavarez, J. Hybrid trait speciation and Heliconius

520 butterflies. Philos. Trans. R. Soc. B Biol. Sci. 363, 3047-3054 (2008).

521 21. Leducq, J.-B. et al. Speciation driven by hybridization and chromosomal plasticity in a wild

522 yeast. Nat. Microbiol. 1, 15003 (2016).

523 22. Roos, C., Kothe, M., Alba, D. M., Delson, E. \& Zinner, D. The radiation of macaques out of Africa:

524 Evidence from mitogenome divergence times and the fossil record. J. Hum. Evol. 133, 114-132

525 (2019).

526 23. Stevison, L. S. \& Kohn, M. H. Divergence population genetic analysis of hybridization between

527 rhesus and cynomolgus macaques. Mol. Ecol. 18, 2457-2475 (2009).

528 24. Fooden, J. Male external genitalia and systematic relationships of the Japanese macaque

529 (Macaca fuscata Blyth, 1875). Primates 12, 305-311 (1971).

530 25. Gompert, Z., Fordyce, J. A., Forister, M. L., Shapiro, A. M. \& Nice, C. C. Homoploid Hybrid

$531 \quad$ Speciation in an Extreme Habitat. Science 314, 1923-1925 (2006).

532 26. Schumer, M., Rosenthal, G. G. \& Andolfatto, P. How Common Is Homoploid Hybrid Speciation?

533 Evolution 68, 1553-1560 (2014).

534 27. Schumer, M., Rosenthal, G. G. \& Andolfatto, P. What do we mean when we talk about hybrid

$535 \quad$ speciation? Heredity 120, 379 (2018).

536 28. Nieto Feliner, G. et al. Is homoploid hybrid speciation that rare? An empiricist's view. Heredity

$537 \quad$ 118, 513-516 (2017). 
29. Fooden, J. Complementary Specialization of Male and Female Reproductive Structures in the Bear Macaque, Macaca arctoides. Nature 214, 939-941 (1967).

30. Fooden, J. Classification and Distribution of Living Macaques (Macaca Lacepede, 1799). in The Macaques: Studies in Ecology, Behavior, and Evolution (ed. Lindburg, D. G.) 1-9 (Van Nostrand Reinhold Company, 1980).

31. Kulathinal, R. J., Stevison, L. S. \& Noor, M. A. F. The genomics of speciation in Drosophila: diversity, divergence, and introgression estimated using low-coverage genome sequencing. PLoS Genet. 5, e1000550 (2009).

32. Green, R. E. et al. A draft sequence of the Neandertal genome. Science 328, 710-722 (2010).

33. Martin, S. H., Davey, J. W. \& Jiggins, C. D. Evaluating the Use of ABBA-BABA Statistics to Locate Introgressed Loci. Mol. Biol. Evol. 32, 244-257 (2015).

34. Martin, S. General tools for genomic analyses. Contribute to simonhmartin/genomics_general development by creating an account on GitHub. (2019).

35. Malinsky, M. et al. Genomic islands of speciation separate cichlid ecomorphs in an East African crater lake. Science 350, 1493-1498 (2015).

36. Martin, S. H. \& Belleghem, S. M. V. Exploring Evolutionary Relationships Across the Genome Using Topology Weighting. Genetics 206, 429-438 (2017).

37. Alby, C. et al. Mutations in KIAA0586 Cause Lethal Ciliopathies Ranging from a Hydrolethalus Phenotype to Short-Rib Polydactyly Syndrome. Am. J. Hum. Genet. 97, 311-318 (2015).

38. Gupta, S., Stamatoyannopoulos, J. A., Bailey, T. L. \& Noble, W. S. Quantifying similarity between motifs. Genome Biol. 8, R24 (2007).

39. Rouillard, A. D. et al. The harmonizome: a collection of processed datasets gathered to serve and

561 40. Lochab, A. K. \& Extavour, C. G. Bone Morphogenetic Protein (BMP) signaling in animal reproductive system development and function. Dev. Biol. 427, 258-269 (2017). 
563 41. Yoshino, T. \& Saito, D. Epithelial-to-mesenchymal transition-based morphogenesis of dorsal

564 mesentery and gonad. Semin. Cell Dev. Biol. 92, 105-112 (2019).

565 42. Yoshino, T., Murai, H. \& Saito, D. Hedgehog-BMP signalling establishes dorsoventral patterning

566 in lateral plate mesoderm to trigger gonadogenesis in chicken embryos. Nat. Commun. 7, 1-11

567 (2016).

568 43. Chang, W. et al. Bmp4 Is Essential for the Formation of the Vestibular Apparatus that Detects

569 Angular Head Movements. PLoS Genet. 4, (2008).

570 44. Nie, X., Xu, J., El-Hashash, A. \& Xu, P.-X. Six1 regulates Grem1 expression in the metanephric

571 mesenchyme to initiate branching morphogenesis. Dev. Biol. 352, 141-151 (2011).

572 45. Lai, D. \& Yang, X. BMP4 is a novel transcriptional target and mediator of mammary cell

573 migration downstream of the Hippo pathway component TAZ. Cell. Signal. 25, 1720-1728

$574 \quad$ (2013).

575 46. Taylor, S. P. et al. Mutations in DYNC2LI1 disrupt cilia function and cause short rib polydactyly

576 syndrome. Nat. Commun. 6, 7092 (2015).

577 47. Zhao, Y. et al. C-terminal Domain (CTD) Small Phosphatase-like 2 Modulates the Canonical Bone

$578 \quad$ Morphogenetic Protein (BMP) Signaling and Mesenchymal Differentiation via Smad

579 Dephosphorylation. J. Biol. Chem. 289, 26441-26450 (2014).

580 48. Kakiuchi, C., Ishiwata, M., Hayashi, A. \& Kato, T. XBP1 induces WFS1 through an endoplasmic

581 reticulum stress response element-like motif in SH-SY5Y cells. J. Neurochem. 97, 545-555

$582 \quad$ (2006).

583 49. Kuroyanagi, H. Fox-1 family of RNA-binding proteins. Cell. Mol. Life Sci. 66, 3895 (2009).

584 50. Zinner, D., Arnold, M. L. \& Roos, C. The strange blood: Natural hybridization in primates. Evol.

$585 \quad$ Anthropol. Issues News Rev. 20, 96-103 (2011).

586 51. Yan, G. et al. Genome sequencing and comparison of two nonhuman primate animal models, the

587 cynomolgus and Chinese rhesus macaques. Nat. Biotechnol. 29, 1019-1023 (2011). 
52. Roos, C. et al. An Updated taxonomy and Conservation Status Review of Asian Primates. Asian Primates J. Vol. $412-38$ (2014).

53. Melnick1, D. J. \& Kidd, K. K. Genetic and evolutionary relationships among Asian Macaques. Int. J. Primatol. 6, 123-160 (1985).

54. Roos, C. Complete mitochondrial genome of a Toque Macaque (Macaca sinica). Mitochondrial DNA Part B 3, 182-183 (2018).

594 55. Exome sequences of toque macaques (Macaca sinica) of Sri Lanka reveal many amino acid changes | bioRxiv. https://www.biorxiv.org/content/10.1101/740761v1.

56. Schumer, M., Cui, R., Powell, D. L., Rosenthal, G. G. \& Andolfatto, P. Ancient hybridization and genomic stabilization in a swordtail fish. Mol. Ecol. 25, 2661-2679 (2016).

57. Scally, A. et al. Insights into hominid evolution from the gorilla genome sequence. Nature 483, $169-175$ (2012).

58. Hibbins, M. S. \& Hahn, M. W. The Timing and Direction of Introgression Under the Multispecies Network Coalescent. Genetics 211, 1059-1073 (2019).

59. Sissler, M., González-Serrano, L. E. \& Westhof, E. Recent Advances in Mitochondrial AminoacyltRNA Synthetases and Disease. Trends Mol. Med. 23, 693-708 (2017).

60. Osada, N. \& Akashi, H. Mitochondrial-nuclear interactions and accelerated compensatory evolution: evidence from the primate cytochrome C oxidase complex. Mol. Biol. Evol. 29, 337-

61. Hill, G. E. et al. Assessing the fitness consequences of mitonuclear interactions in natural populations. Biol. Rev. 94, 1089-1104 (2019).

62. Glasgow, R. I. C. et al. Novel GFM2 variants associated with early-onset neurological presentations of mitochondrial disease and impaired expression of OXPHOS subunits. Neurogenetics 18, 227-235 (2017).

63. Liang, L.-P. et al. Mitochondrial oxidative stress and epilepsy in SOD2 deficient mice: 
attenuation by a lipophilic metalloporphyrin. Neurobiol. Dis. 45, 1068-1076 (2012).

614 64. Trier, C. N., Hermansen, J. S., Sætre, G.-P. \& Bailey, R. I. Evidence for Mito-Nuclear and Sex-

615 Linked Reproductive Barriers between the Hybrid Italian Sparrow and Its Parent Species. PLOS Genet. 10, e1004075 (2014).

65. Meiklejohn, C. D. et al. An Incompatibility between a Mitochondrial tRNA and Its NuclearEncoded tRNA Synthetase Compromises Development and Fitness in Drosophila. PLOS Genet. 9, e1003238 (2013).

66. Ellison, C. K. \& Burton, R. S. Cytonuclear conflict in interpopulation hybrids: the role of RNA polymerase in mtDNA transcription and replication. J. Evol. Biol. 23, 528-538 (2010). 1509-1511 (2017).

68. ncbi/sra-tools. (NCBI - National Center for Biotechnology Information/NLM/NIH).

69. Li, H. Toolkit for processing sequences in FASTA/Q formats: lh3/seqtk. (2019).

70. Martin, S. H., Davey, J. W., Salazar, C. \& Jiggins, C. D. Recombination rate variation shapes

71. Terhorst, J., Kamm, J. A. \& Song, Y. S. Robust and scalable inference of population history from barriers to introgression across butterfly genomes. PLOS Biol. 17, e2006288 (2019). hundreds of unphased whole-genomes. Nat. Genet. 49, 303-309 (2017).

72. Hinrichs, A. S. et al. The UCSC Genome Browser Database: update 2006. Nucleic Acids Res 34, D590-8 (2006).

73. Mi, H. et al. Protocol Update for large-scale genome and gene function analysis with the

634 74. Waal, F. B. M. de. Peace Lessons from an Unlikely Source. PLOS Biol. 2, e101 (2004).

635 75. Danecek, P. et al. The variant call format and VCFtools. Bioinformatics 27, 2156-8 (2011).

636 76. Conway, J. \& Gehlenborg, N. UpSetR: A More Scalable Alternative to Venn and Euler Diagrams for 637 Visualizing Intersecting Sets. (2019). 
Figure 1. Summary of focal species, Macaca arctoides, its evolutionary relationships, and the data used. (a) $M$. arctoides has the largest baculum among primates and is a putative homoploid hybrid species. Image "Stumptail Monkeys" from ${ }^{74}$ by Frans de Waal licensed under CC. (b) Model based on literature 10,14,15 depicts possible hybrid speciation between sinica and fascicularis species groups to form $M$. arctoides, with split time uncertainty relative to split of fascicularis group species, M. mulatta and $M$. fascicularis ${ }^{22}$. Approximate split times from ref ${ }^{15}$. This study included WGS from $M$. arctoides and representative members of each species group. Baboon was used as an outgroup species. (c) Geographic range of M. arctoides and other focal species in this study. M. arctoides has present day geographic overlap with members of both species groups (redrawn from ${ }^{30}$ ). Background image "Topographic map of East Asia" by Ksiom is licensed under CC BY-SA 3.0. (d) Intersection of heterozygous sites between the five species. The output of vcf-compare from vcftools ${ }^{75}$ was input into the UpSetR package ${ }^{76}$ to plot the intersection of sites. Values in the left plot match count of heterozygous sites per sample in Table 1. (e) Inferred recent effective population size trajectories for each species using smc++. Years before present based on a generation time of 6 years per generation. arc - M. arctoides, fas - M. fascicularis, mul - M. mulatta, thi - M. thibetana, ass - M. assamensis. (f) Topology weight distribution of three rooted topologies of species relationships in sliding windows 656 along the genome. bab - P. anubis.

a

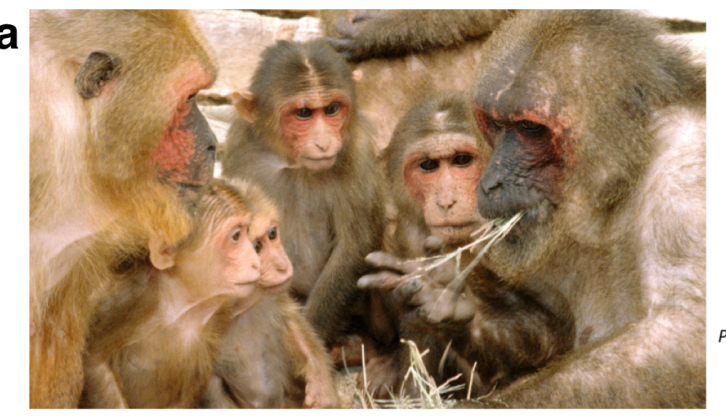

C

657

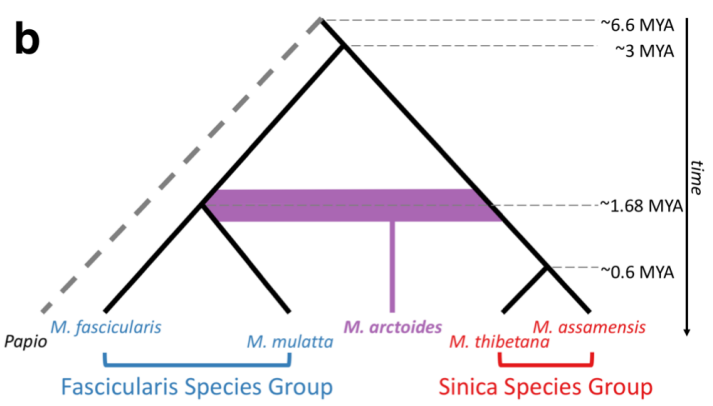

d
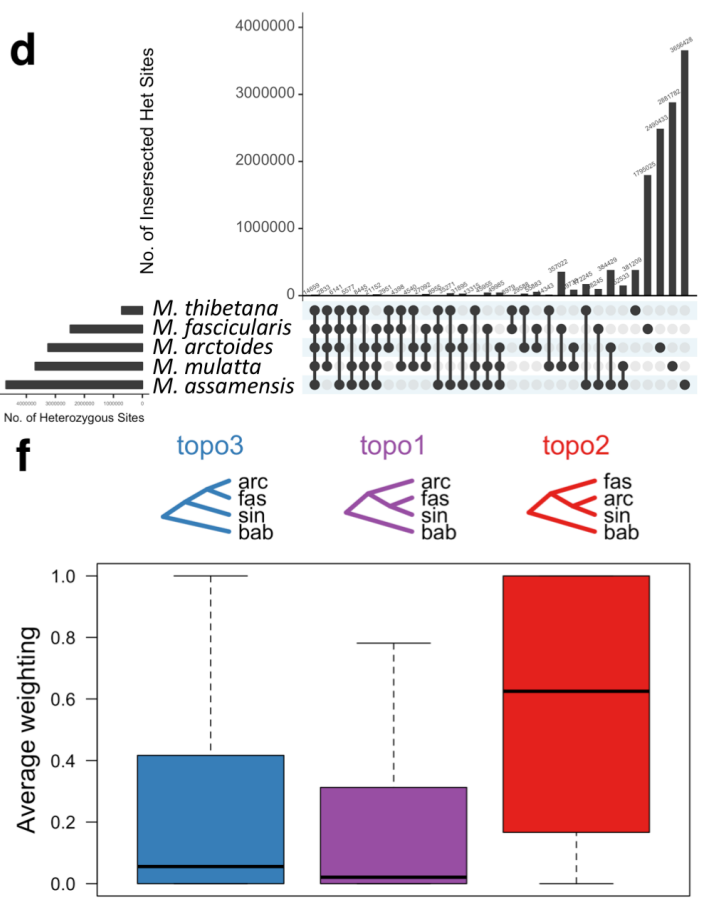
bioRxiv preprint doi: https://doi.org/10.1101/2020.05.18.102251; this version posted May 20, 2020. The copyright holder for this preprint (which

was not certified by peer review) is the author/funder, who has granted bioRxiv a license to display the preprint in perpetuity. It is made available under aCC-BY-ND 4.0 International license.

Figure 2. Introgression analysis. Results from analysis of introgression $\left(f_{d M}\right)$ in $50 \mathrm{~kb}$ sliding windows (a) using all three $M$. arctoides samples, (b) only the Malaya sample, (c) only the SM1 sample, and (d) only the SM2 sample. Regions where M. arctoides has most recent ancestry with the sinica group are displayed as negative values and regions where M. arctoides shares most recent common ancestry with the fascicularis group are displayed as positive values. Other window size

664
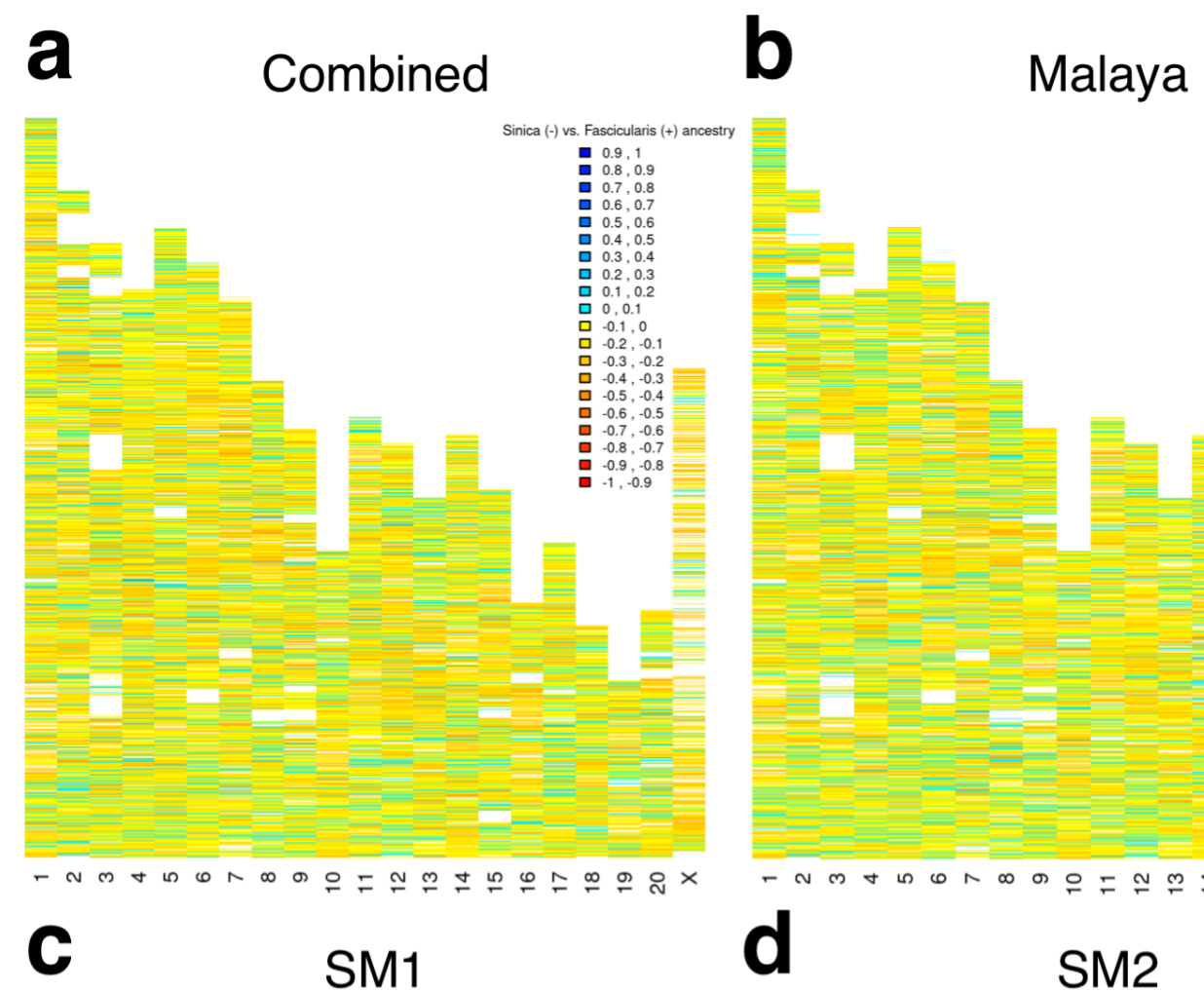

\section{SM1}
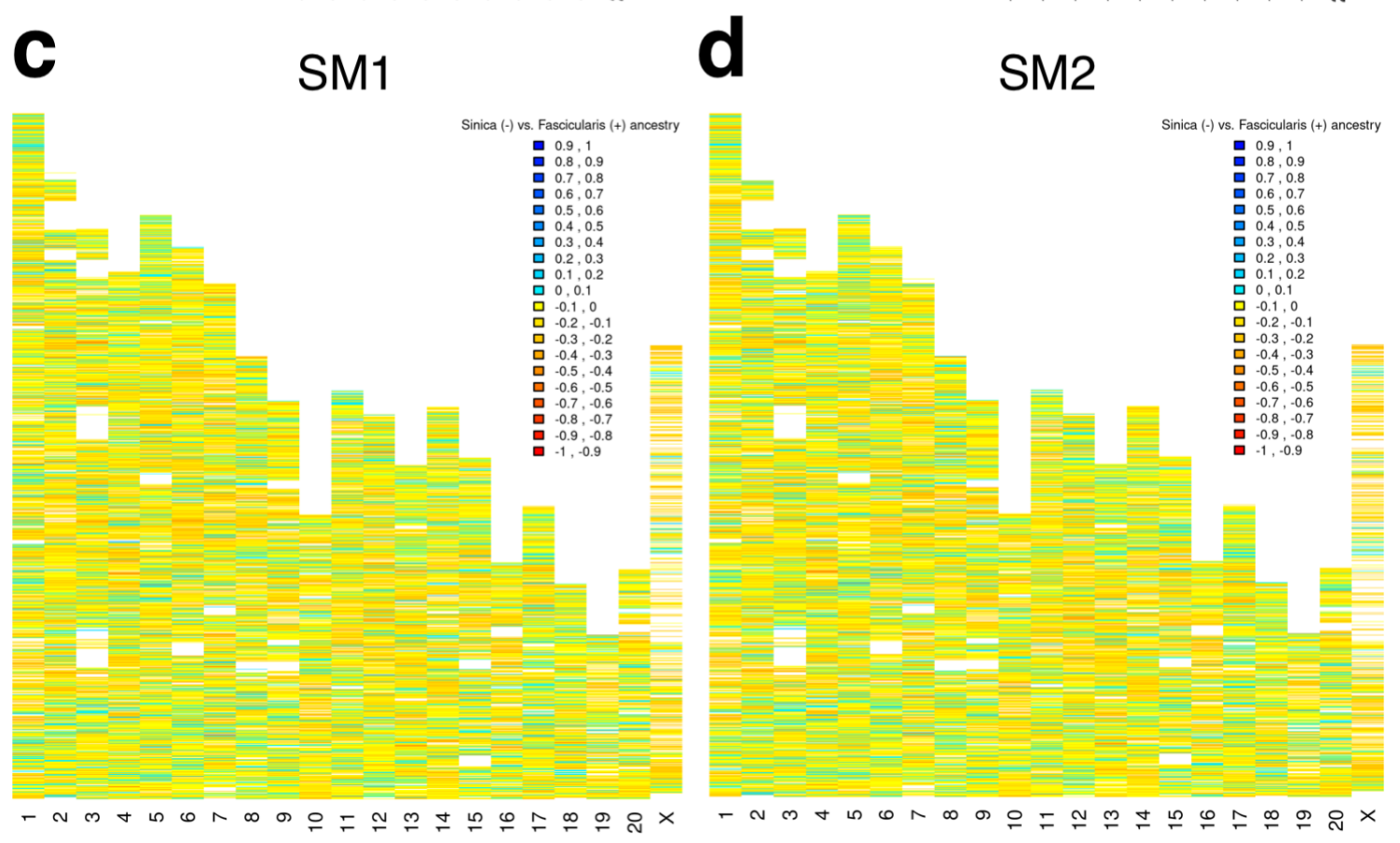
Figure 3. Predicted TF binding motifs in outlier genes across all three possible gene topologies. Fixed differences were examined for predicted differential TF binding. (a) Genes overlapping majority topo1 regions. KIF14, which overlaps baculum QTL region \#1 shows unique binding of CTCF for the M. arctoides allele. (b) Genes overlapping majority topo2 regions. CTDSPL2 is a sinica origin outlier gene with a $f_{d M}$ score of -0.82. (c) Genes overlapping majority topo3 regions. BMP4, which overlaps baculum QTL region \#2 has two SNPs in the 3'UTR that have differential predicted binding to two proteins known to interact with BMP4. Literature predicts that both of these changes would increase BMP4 expression in M. arctoides as compared to other species. DYNC2LI1 is a fascicularis origin outlier gene with a $f_{d M}$ score of 0.79 and is known to interact with BMP4.

a

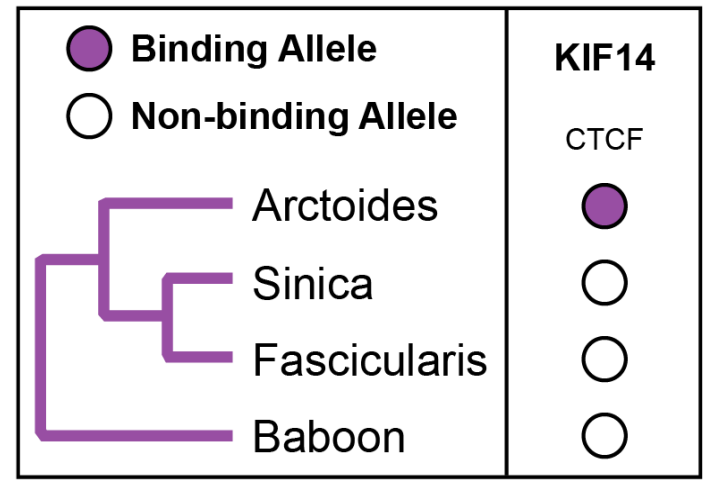

b

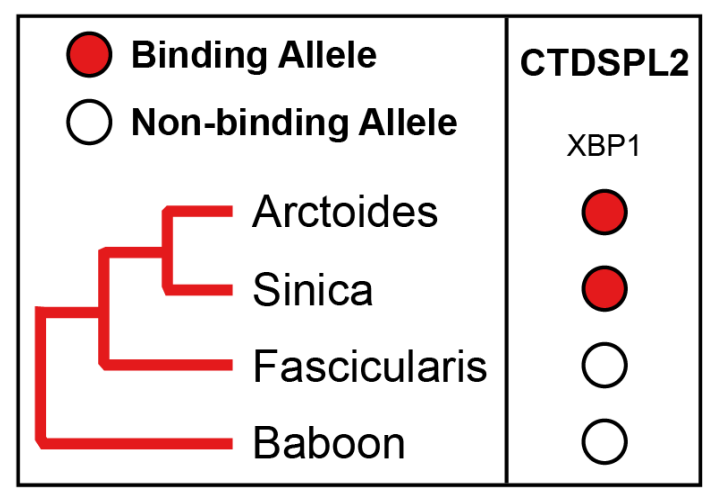

C

\begin{tabular}{|c|cc|c|}
\hline Binding Allele & \multicolumn{2}{|c|}{ BMP4 } & DYNC2LI1 \\
$\bigcirc$ Non-binding Allele & SIX1 & TEAD1/3 & RBFOX2 \\
Arctoides & $\bigcirc$ & $\bigcirc$ & $\bigcirc$ \\
Fascicularis & $\bigcirc$ & $\bigcirc$ & $\bigcirc$ \\
Sinica & $\bigcirc$ & $\bigcirc$ & $\bigcirc$ \\
Baboon & $\bigcirc$ & $\bigcirc$ & $\bigcirc$ \\
\hline
\end{tabular}


bioRxiv preprint doi: https://doi.org/10.1101/2020.05.18.102251; this version posted May 20, 2020. The copyright holder for this preprint (which

was not certified by peer review) is the author/funder, who has granted bioRxiv a license to display the preprint in perpetuity. It is made available under aCC-BY-ND 4.0 International license.

677 Figure 4. Gene ontology results. Gene ontology results from genes with majority topo1 (purple in 678 Figure 1F). Results obtained using PANTHER GO-Slim Biological Process. 4,072 IDs were used 679 resulting in a total of 6,427 process hits.

680

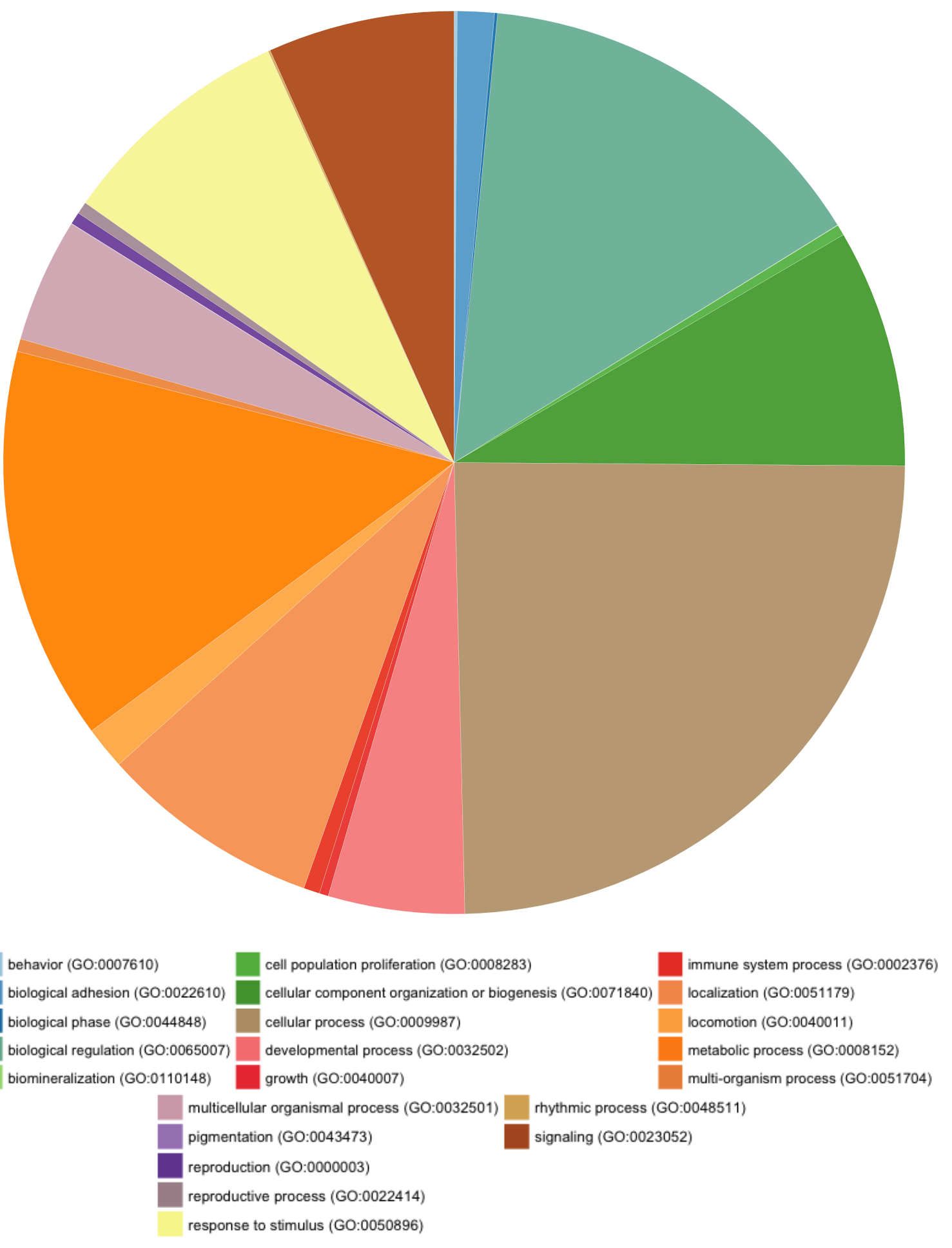


685 Table 1. Sample information. A list of samples used in this study with a summary of genome 686 information. Two samples were sequenced as part of this study (in bold) and the remainder were 687 downloaded from NCBI.

688

\begin{tabular}{|c|c|c|c|c|c|c|c|c|}
\hline ID & species & $\begin{array}{l}\text { Species } \\
\text { Group }\end{array}$ & Coverage & $\begin{array}{c}\text { No. sites } \\
\text { VQSR Passed }\end{array}$ & $\begin{array}{c}\text { No. sites } \\
\text { heterozygous }\end{array}$ & $\begin{array}{c}\text { NCBI } \\
\text { Accession }\end{array}$ & SAMN_ID & Sex \\
\hline Malaya & M. arctoides & arctoides & 20 & $6,849,957$ & $3,252,204$ & SRS6488501 & 14521207 & $\mathbf{F}$ \\
\hline SM1 & M. arctoides & arctoides & 32 & $6,848,826$ & $3,251,892$ & SRR2981139 & 04316319 & $\mathrm{~F}$ \\
\hline SM2 & M. arctoides & arctoides & 19 & $6,833,185$ & $3,246,931$ & SRR2981140 & 04316320 & $\mathrm{~F}$ \\
\hline BGI-CE-4 & M. fascicularis & fascicularis & 43 & $4,547,767$ & $2,486,762$ & SRS114988 & 00116341 & $\mathrm{~F}$ \\
\hline CR-5 & M. mulatta & fascicularis & 44 & $4,561,581$ & $3,693,024$ & SRS115022 & 00113489 & $\mathrm{~F}$ \\
\hline $\mathrm{BGI}$ & M. mulatta & fascicularis & 10 & $4,531,187$ & $3,678,452$ & SRS212016 & 00627571 & $M$ \\
\hline BGI-96346 & M. mulatta & fascicularis & 4 & $4,334,465$ & $3,533,580$ & SRS114988 & 00113455 & M \\
\hline A20 & M. assamensis & sinica & 13 & $7,567,951$ & $4,680,934$ & SRS6491954 & 14521608 & $\mathbf{F}$ \\
\hline $\mathrm{XH} 1$ & M. assamensis & sinica & 49 & $7,669,854$ & $4,702,055$ & SRR2981114 & 04316321 & $M$ \\
\hline $\begin{array}{l}\text { Tibetan- } \\
\text { macaque- } \\
\text { NO.3 }\end{array}$ & M. thibetana & sinica & 27 & $4,844,002$ & 713,449 & SRR1024051 & 02390221 & $\mathrm{~F}$ \\
\hline
\end{tabular}

